\title{
Mesure de salinité
}

\section{Salinity measurements}

\author{
PAR MV. SAUYAGE DE SAINT-MARC ET BOUDAN \\ Ingéneurs aU Labohatome Datphinols d'Hymaudoue (Neyrpic)
}

\section{English synopsis p. 217}

L'océanographe n'est pas seul à être intéressé par la salinité de l'cau; l'hydraulicien aussi, dans certains problemes, a besoin de comaitre cette caractéristique de l'eau; tels sont les problèmes des estuaires (courants de densité, pénétration de la langue d'eau salée à l'intérieur

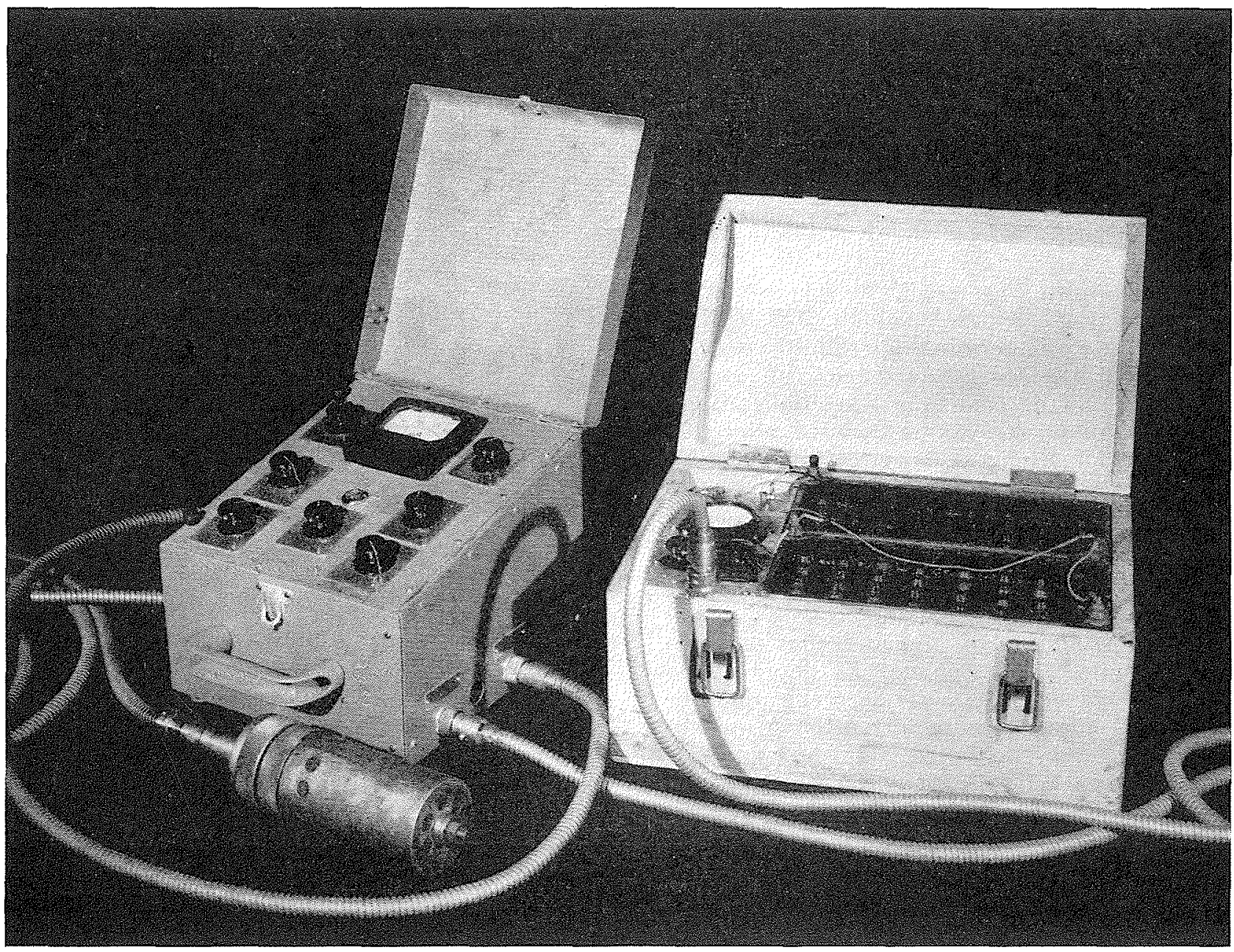

Le HaLomètre. - La sonde, les accumulateurs d'alimentation et le cadran de commande et de lecture. 
d'un estuaire, évolution du fond des estuaires et de leur voisinage, etc...) ou encore les problemes d'identification des nappes soutermaines salées.

Mais alors que l'océanographe opère sur une bande très étroite de salinité (autour de $30 \mathrm{~g} / \mathrm{kg}$ d'eau, l'hydraulicien souhaite couvrir une gamme très large (de 0 à $35 \mathrm{~g} / \mathrm{kg}$ d'eau). De plus, ce dernier est fort intéressé par la connaissance du gradient de densité; il lui faut done pouvoir tracer la variation de salinité en fonction de la profondeur, et comme cette salinité peut parfois varier dans une très large mesure sur une profondeur faible (de l'ordre de 5 à $10 \mathrm{~cm}$ ), l'hydraulicien désire également procéder à des mesures presque ponctuelles.

Après avoir précisé aussi les exigences de l'hydraulicien relatives à la mesure de la salinité, les auteurs passent en revue les différents appareils existant en essayant de les classer à la fois suivant le mode opératoire et suivant le principe même mis en jeu pour la mesure. Et comme aucun de ces appareils ne répondait aux exigences précitées, le Laboratoire Dauphinois d'Hydraulique en étudia et en mit au point un nouveau désigné sous le nom de « halomètre ».

\section{LE HALOMETTRE}

\section{1. - Principe de l'appareil.}

Le halomètre détermine la salinité in situ par la mesure de la conductance d'une sonde plongée au sein de l'eau que l'on étudie. L'eau salée étant un électroytc, le courant alternatif est choisi pour alimenter les circuits de mesure afin d'éviter l'électrolyse et l'apparition de forces électromotrices parasites au contact des électrodes et de la solution.

Le principe de la mesure de conductance est représenté par la figure 1.

Une source alternative alimente, sous une tension constante $U$, l'impédance $Z_{3}$ de la sonde et une impédance $Z_{2}$ en série avec elle. Un voltmètre électronique mesure la tension $u$ qui apparaît aux bornes de $Z_{2}$.

Cette impédance $Z_{2}$ est choisie de manière qu'elle soit pratiquement en quadrature avec $Z_{3}$ et que son module soit inférieur au quinzième de la plus faible valeur que prend le module de $Z_{3}$. Dans ces conditions, le module de l'impédance série $Z=Z_{2}+Z_{3}$ est égal au module de l'impédance à mesurer à $0,2 \%$ près, ce qui se traduit par $Z \# Z_{3}$.
Le courant absorbć par le voltmètre chant négligeable devant le courant traversant $Z_{2}$ et $Z_{3}$, la valeur de courant I est domice par :

$$
\mathrm{I}=\frac{\mathrm{U}}{\mathrm{Z}} \# \frac{\mathrm{U}}{Z_{3}}
$$

La tension aux bornes de $Z_{2}$ est :

$$
\mathrm{u}=Z_{2} \mathrm{I}=\frac{Z_{2} \mathrm{U}}{Z_{3}}=\mathrm{AU}_{\gamma}
$$

$\gamma$ est la conductance de la sonde, l'argument de $Z_{3}$ étant en effet très faible. A est une constante.

La puissance dalimentation varic suivant les valeurs de $Z_{3}$; ceci nécessite une stabilisation de

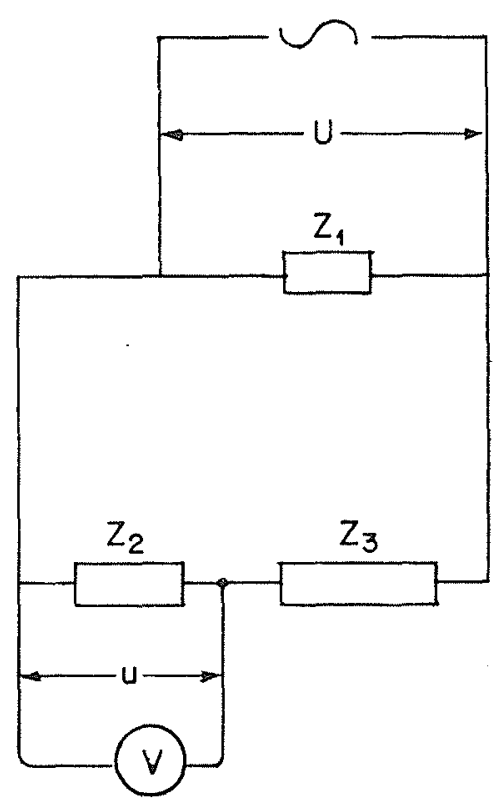

FIG. 1.

la tension $U$, stabilisation qui est obtenue au moyen d'une source de faible impédance interne, shuntée par une impédance de grand module devant celui de l'impédance à mesurer et en quadrature avec elle.

Cependant, cette simple mesure ne suffit pas; en effet, la conductivité du liquide de la sonde est fonction également de la température; or, cette température varie d'un point à un autre au sein de la mer ou de la nappe souterraine; on s'arrange donc à éliminer de la mesure l'influence de ces variations de température par le dispositif indiqué ci-après et désigné sous le nom de compensateur. 


\section{Compensation des variations de tempé- rature.}

La compensation est réalisée en asservissant la tension d'alimentation $U$ aux variations de température. C'est pour cela que cette tension U est prise aux bornes d'une sonde $Z_{\theta}$ (sonde de compensation) de salinité fixe et dont la température est égale à colle de $Z_{3}$ (fig. 2).

Le compensateur est alimenté à courant constant sous une tension $U_{1}$. Cette alimentation est réalisée par la mise en série avec $Z_{c}$ d'une impédance $Z_{b}$ en quadrature avec elle et dont le module est supéricur à quinze fois le module maximum de $Z_{c}$; la variation d'impédance de la sonde

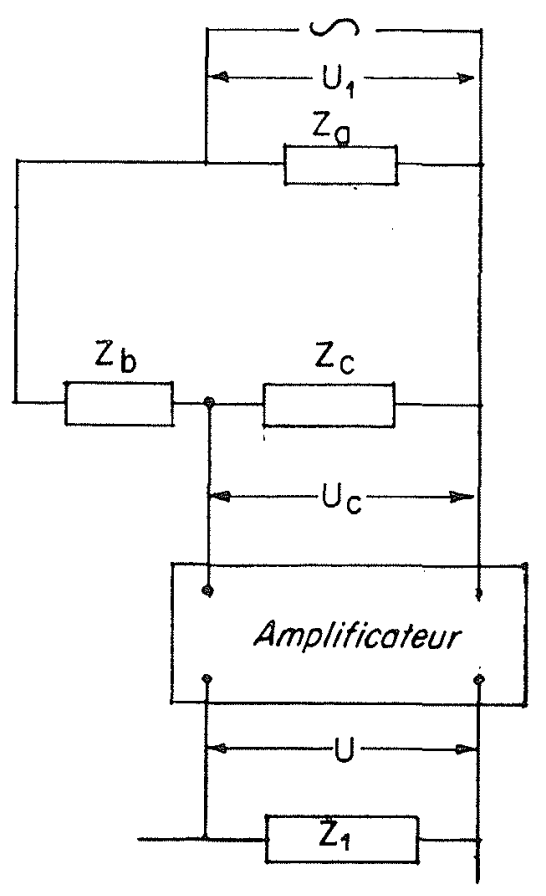

FIG. 2.

$Z_{*}$ n'étant pas de $100 \%$, l'alimentation à courant constant est réalisée à moins de $0,2 \%$ près.

La conductance est une fonction de la forme :

$$
y=f(s) \oplus(\theta)
$$

$s$ est lat salinité,

o la température.

La tension $\mathrm{U}_{e}$ aux bornes de $Z_{c}$ est donnée par :

$$
\mathrm{U}_{c}=\mathrm{B} \mathrm{Z} \mathrm{Z}_{1}
$$

En remplaçant $Z_{*}$ par sa valeur :

$$
Z_{c}=\frac{1}{\gamma_{c}}=\frac{1}{f\left(s_{0}\right) \oplus(\theta)} ;
$$

il vient :

$$
\mathrm{U}_{c}=\frac{\mathrm{BU}_{1}}{f\left(s_{c}\right) \oplus(0)}
$$

La tension $U_{0}$ est amplifiée par l'amplificateur A, done :

$$
\mathrm{U}=\frac{\mathrm{C} \mathrm{U}_{1}}{f\left(s_{c}\right) \varphi(0)}
$$

C est une constante tenant compte de l'amplification.

En remplacant dans (1) $U$ et $\gamma$ par leurs expressions respectives (3) et (2), on obtient :

$$
u=\frac{\mathrm{FU}_{1} f(s) \varphi(\theta)}{f\left(s_{c}\right) \varphi(\theta)}
$$

d'où :

$$
u=\mathrm{K} f(s)
$$

la lecture est donc indépendante de la température.

Pour connaitre la conduclivité réelle due à la température du point où l'on effectue la mesure, il suffit de remplacer la sonde $Z_{e}$ par une résistance en constantan dont le coefficient de température est nul.

\section{3. - Mesure de la température.}

En remplaçant la sonde $Z_{3}$ par une sonde de salinité fixe (sonde de température) et la sonde $Z_{c}$ par une résistante en constantan, la tension de sortie n'est alors fonction que de la température.

Cette mesure de température nécessite la précision suivante : la variation de conductance de la sonde de température est moins importante dans la zone de mesure que celle de la sonde de salinité. Pour augimenter la précision, d'une part le gain de l'amplificateur est augmenté et, d'autre part, à la tension de sortie détectée est opposée une tension continue fixe dont la valeur amène l'aiguille de l'appareil au zéro pour la plus basse température. L'échelle des températures couvre ainsi tout le cadran.

\section{4. - Les sondes.}

Une sonde est un bac à électrolyse en courant alternatif; électriquement, c'est une impédance complexe que l'on schématise par une résistance avec une capacité qui peut être soit en série, soit en parallèle. 


\section{ChorX des IMPÉdances}

Les considérations développées an sujet des valeurs respectives des impédances $Z_{2}$ et $Z_{3}$ d'une part, $Z_{l}$ et $Z_{e}$ d'autre part, régissent le choix des impédances. Ainsi, l'impédance d'une sonde ne peut varier qu'entre une limite inférieure et une limite supérieure; et le rapport des valeurs des impédances correspondant à ces limites extrêmes étant assez faibles, trois sondes sont nécessaires pour couvir toute la gamme des salinités rencontrées (exigence mentionnée au début de cet exposé). A chaque sonde correspond une sensibilité; les trois sensibilités obtenues portent a 12 le rapport entre les mesures extremes effectuées par le halomètre.

\section{STABILITÉ DES SONDES}

L'impédance des sondes dépend de nombreux facteur's et notamment clle varie avec le temps: c'est le vieillissement des̀ sondes. Ce vieillissement, qui se traduit par une augmentation d'impédance, est fonction surtout de la nature du métal des électrodes et de la densité de courant au voisinage de ces dernières.

Le métal adopté dans la réalisation des électrodes est un acier inoxydable au nickel chrome, soigneusement poli.

La densité de courant est diminuée au voisinage des électrodes par une forme gémétrique des sondes appropriées.

\section{GÉométrie des sondes}

La sonde classique biplaque à champ libre ne permet pas de réaliser en eau chargée, pour des dimensions géométriques raisonnables, des densités de courant sur les plaques conciliables avec la stabilité, ni les valeurs d'impédances justifiant les approximations faites. Pour satisfaire à ces conditions, une sonde à électrode unique a été réalisée.

Ainsi, la conductance mesurée est bien déterminée, la conductance du milien liquide cntre la sortie du tube et la masse étant négligeable.

\section{5. - Tarage et vérification de l'appareil. Tarage de la mesure de conductivité.}

Comme $u=k$ ky (1), il suffit, pour tarer l'appareil, d'ajuster la pente de la droite d'étalonnage. Un commutateur est amené sur la position « tarage de conductivité $»$ la sonde de mesure de salinité et la sonde de compensation sont alors remplacées par des résistances connues. L'ai- guille est amenée sur la position correspondant à la valeur de tarage au moyen d'un potentiometre dit de tarage de conductivite. Ceci suppose que la linéarité est bonne; cette linéarité est vérifice en mettant lo commutateur sur la position « vérification, linéarité » qui remplace la résistance connue correspondant au compensateur par une résistance de valeur moitié.

\section{ADAPTATION DU COMPENSATEUR}

'Trois types de compensateur's sont utilisés; it chacun d'eux correspond une certaine gamme de salinité.

Le compensateur choisi ćtant en place sur l'appareil, un commutateur est mis sur la position "adaptation compensateur » qui branche le compensateur et remplace la sonde en constantan. A l'aide d'un potentiomètre, le gain de l'amplificateur de sortic du compensateur est modifié pour amener l'aiguille à la position correspondant à la valeur du tarage.

L'appareil est prêt pour une campagne de mesure, campagne au cours de laquelle la commodité du tarage permet une vérification rapide des bonnes conditions de marche de l'appareil.

Les auteurs terminent en signalant que cet appareil a déjà reç avee succès l'épreuve de l'expérience.

\section{I S C US SION}

M. le Plésident remereie M. SAUvage de SANT-Mare, souligne la clarte de son exposé ainsi que l'interet des questions de salinite en mer et de l'appareil présenté.

Sul une question de M. Brav, relative a l'utilisation de l'appareil dans les grandes profondeurs $(200-300 \mathrm{~m})$, M. SaUvake répond que eetle question n'est posée pour le Laboratoire Dauphinois que pour des profondeurs de 100 à $150 \mathrm{~m}$ au maximum (estuaires), mais gue son adaptation aux grandes profondeurs consisterat seulement at étudier plus à fond la tenue des appareils de desecnte.

Sur des questions de M. Lacombl: ot de M. Bonix relatives aux difficultés crées par l'isolement, les effets de capacite et les parasites, M. SAUvage DE SANT-Mare: dit que ces effets existent mais sont mieux compensés dans l'appareil présenté que dans le pont de Knudsen; quo d'autre part, les sondages pratiqués jusqu'à présent nont pas pose la question de la profondeur limite due a la capacité du câble.

M. Dunano explique que le Taboratoire Dauphinois d'Hydraulique utilise, en dehors de cet appareil simplitic pour les mesures dans la nature, d'autres sondes du mène type mais comportant des perfectionnements électrigues ou electroniques pour l'analyse des concentralions spatiales en mixtures, bulles d'air, etc.

Sollicité par M. Savvage ne SanT-Manc et pat M. Ie Président, M. LACome donne un aperesu de l'appareil américain S.T.D. concu dans un but analogue. Cet appareil compliqué et de grandes dimensions, mesure da fois la tempémature, la profondeur, la salinité et la densité, il a done quatre asservissements et un rattrapage automatique de l'équilibre des ponts; la sonde est petite, 
par contre, lappareil a servi dans les estuaires, mais non pas pour les mesures oceanographiques, pour lesquelles sa précision, comme celle de lappareil présenté, seraient insuffisantes (dix fois moins sensibles que la méthode internationale de linudsen).

Sur une nouvelle question de M. Bonnin, M. Sauvate DE Sant-Marc répond que le critère d'homogénéité de la densité de l'eau dont on mesure la salinité, se fait par la comparaison des trois mesures, qu'il a signalées; le cas échéant, on fait un nouveau prélèvement d'eau pour la sonde-témoin.

N. Hupser demande au conférencier si un appareil de ce genre peut ètre rendu sufisamment robuste pour ètre employe sur les chantiers de certains barages pour lesquels il est utile de commaitre la salinité des caux circulant dans les terrains de fondation.

M. SaUvage de Sant-Marc répond que la nouvello sonde est conçue pour aller dans des sondages de 40 ì $50 \mathrm{~cm}$ de diamètre, et que c'est simplement l'encombrement de l'appareil qui limite la profondeur des sondages.
M. le Président remercic M. Hurnen et présente deux remarques sur certaines expressions contenues dans l'exposé :

1. La loi de la constance de la constitution de l'eau de mer n'aurait pas été trouvée par KNudsen (voil 'Traité d'Ccéanographie (e Rouch);

20 La salinité s'exprime en grammes de sel par kilo d'eat, pour tenir compte de l'influence avec la température, et de préférence en millièmes, do telle sorte que la précision des mesures se chiffre en fraction de nillièmes (par exemple $35,5 \%$ ).

M. Ie Président souligne un des avantages de l'appareil présenté, qui consiste à domner la salinité in situ et avec un bagage peu encombrant. Son emploi semble bien limité aux estuaires, mais d'une manière générale, les mesures de salinité au large offrent assez peu d'intérêt et les autres systèmes employés en océanographie no donnent pas grand'chose.

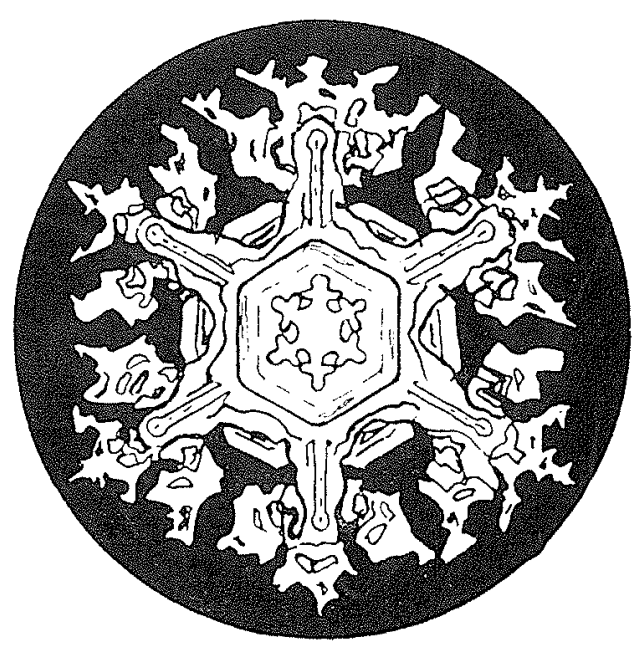

